\title{
Gestational diabetes mellitus: from thought to action
}

\author{
Bharti Kalra $\cdot$ Sanjay Kalra
}

Received: 11 October 2013 / Accepted: 6 December 2013 / Published online: 22 January 2014

(C) Research Society for Study of Diabetes in India 2014

"If you can't measure it, you can't improve it" Lord Kelvin

Lord Kelvin's statement rings true today in medical practice, as in temperature measurement. The sine qua non for improving clinical outcomes is better clinical practice. To improve that, we first need to measure it [1]. Nowhere is this more important than in gestational diabetes mellitus (GDM) which is insidious, common and hard to detect. Much depends on physicians and obstetricians caring for pregnant women to be aware of the principles in screening and management of GDM. Data are hard to come by, especially from India. Therefore, the study reported in this issue of the Journal by Gupta et al. is welcome in that it provides a benchmark in the management of GDM [2]. Carried out in Bhatinda, Punjab, Gupta et al. utilize a structured questionnaire to assess current clinical practices of obstetricians and physicians. It covers various practices related to prevention, detection and management of GDM.

Gestational diabetes mellitus (GDM) is a distinct entity, similar to, yet different from, type 2 diabetes mellitus (T2DM). Diagnosed during pregnancy, it has unique screening, diagnostic and therapeutic modalities. Its pathophysiologic features overlap those of T2DM, but are not exactly the same. This is true for its diagnosis and treatment as well [3]. All physicians however, are not necessarily aware of these differences. All obstetricians, too, who manage the majority of women with GDM, may not be up dated with current trends in GDM care.

This is unfortunate, because suboptimal GDM management adversely impacts both material and foetal health, and may influence the development of metabolic disease when the newborn reaches adulthood.

Guidelines and recommendations are available addressing the prevention, detection and management of GDM. The

B. Kalra $(\bowtie) \cdot$ S. Kalra

Bharti Hospital, Kunjpura Road, Karnal 132001, India

e-mail: brideknl@gmail.com multidisciplinary nature of GDM care, and persistent controversy regarding diagnostic criteria and therapeutic choices $[4$, 5], however, mean that actual clinical care may be non uniform across regions, health care facilities, and even between departments in the same Institution. It is therefore important to study the current trends in screening, diagnosis, management and follow up of GDM by those caring for pregnant women.

The information thus obtained helps set a reference for future studies. It helps diabetes care professionals and bodies to identify lacunae in existing medical knowledge, and plan appropriate continuing medical education (CME) programmes for health care providers. Detailed analysis of the data allows organizers to prepare an audience-specific agenda, suited to the needs of obstetricians and physicians. The data also helps us ponder the factors which create a barrier between current medical research, knowledge, and practice. Identification of these barriers, in turn, can help creation of bridges to address the same.

The results of this study highlight the actual status of medical care at ground level. Coming from a state which does not produce much endocrine or diabetes literature [6], this is a creditable effort to quantify real life clinical praxis. It highlights the need to educate medical professionals about current trends in screening, diagnosis, non-pharmacological management, drug therapy, and follow up of GDM. Its findings, and recommendations, should serve as a wake up call for all stakeholders in diabetology.

Gupta et al. report that practices related to GDM are grossly suboptimal. Universal screening is still a distant dream: only three out of ten physicians and obstetricians practice this, and only two out of ten use an oral glucose tolerance test in high risk antenatal cases. Management strategies, too, are suboptimal, with just eight out of ten using insulin as drug of choice. Surprisingly, neither the authors, nor the respondents, mention medical nutrition therapy (MNT) as a treatment for GDM. Only one out of ten consultants orders glycated hemoglobin 
assessment during treatment, with nine of ten participants suggesting that a blood glucose done once a week or once a fortnight is enough for follow up. Postpartum follow up is recommended by only six of ten consultants, with nearly nine out of ten reporting fasting glucose as the investigation of choice. There is a difference between responses of obstetricians and physicians: this raises the need for interdisciplinary [with effective bidirectional communication between concerned specialties] management of the condition.

These statistics are similar to awareness levels reported among rural antenatal women from Tamil Nadu [7]: in some aspects, these women display a better knowledge of GDM related issues. The data reported by Gupta et al. compares unfavourably with that reported from Australia a decade ago [8]. Though Australian hospitals exhibited marked heterogeneity in their screening practices, the majority did provide adequate care and follow up facilities to women with GDM.

Hopefully, concerted efforts will lead to an improvement in management of GDM. A follow up survey, using validated questionnaires, and appropriate statistical tools, should reveal optimal clinical practices among both obstetricians and physicians, within a few years. This survey should include aspects of nutritional and psychosocial care, apart from the screening and pharmacological management issues studied in the current paper.

Professional organizations such as Research Society for Study of Diabetes in India (RSSDI) should take the lead, to ensure that current guidelines reach every physician and obstetrician. All concerned specialties, including obstetrics, medicine, endocrinology, medical nutrition, and psychology, should be involved in this task. National and state health authorities can contribute by making GDM management an essential part of CMEs for all government and practicing doctors. The same can be done at undergraduate and postgraduate levels by curriculum planners. Similar campaigns are underway in Europe as well [9].
The International Journal of Diabetes in Developing Countries (IJDDC) has supported this movement, by publishing on GDM in the past [10]. It will continue to stimulate research on the challenges faced in GDM management in developing nations. Hopefully, thus will help identify, and utilize, solutions to overcome these challenges. This in turn, will achieve optimal health, both for women with GDM, and for their unborn offspring.

\section{References}

1. Appa Rao A, Sridhar GR. Quality of care: assessment. Lipids Health Dis 2007;6:12

2. Gupta VK, Maria AK, Singh AA, et al. Current practices of prevention, detection \& management of gestational diabetes mellitus in Punjab. Int J Diabetes Dev Ctries. 2013. doi:10.1007/s13410-0130141-3.

3. Gupta Y, Kalra S. Gestational diabetes. J Pak Med Association. 2013;63:1064-8.

4. Kalra S, Malik S, John M. Gestational diabetes mellitus: a window of opportunity. Indian J Endocr Metab. 2011;15:149-51.

5. Magon N, Seshiah V. Gestational diabetes mellitus: non-insulin management. Indian J Endocr Metab. 2011;15:284-93.

6. Kumar KH, Aravinda K. Trends in endocrinology related research articles in a medical journal from India. Indian J Endocr Metab. 2012;16:931-4.

7. Shriraam V, Rani SM, Sathiyasekaran B, et al. Awareness of gestational diabetes mellitus among antenatal women in a primary health center in South India. Indian J Endocr Metab. 2013;17:146-8.

8. Rumbold AR, Crowther CA. Guideline use for gestational diabetes mellitus and current screening, diagnostic and management practices in Australian hospitals. Aust N Z J Obstet Gynaecol. 2001;41:86-90.

9. Buckley BS, Harreiter J, Damm P, et al. Gestational diabetes mellitus in Europe: prevalence, current screening practice and barriers to screening. A review. Diabet Med. 2012;29:844-54.

10. Seshiah V. Clinical scoring in gestational diabetes screening. Int $\mathbf{J}$ Diabetes Dev Ctries. 2012;32:63-4. 